\title{
OCCULT BREAST CARCINOMA PRESENTING WITH UNILATERAL ORBITAL AND CUTANEOUS METASTASES IN A HYPOTHYROID PATIENT
}

Wettasinghe $\mathrm{MC}^{1}$, Weerasinghe $\mathrm{V}^{2}$, Wijetunge $\mathrm{S}^{3}$, Hewavithana $\mathrm{PB}^{1}$

${ }^{1}$ Department of Radiology, Teaching Hospital Peradeniya, Sri Lanka

${ }^{2}$ Department of Physiology, Faculty of Medicine, Peradeniya, Sri Lanka

${ }^{3}$ Department of Pathology, Faculty of Medicine, Peradeniya, Sri Lanka

DOI:[http://doi.org/10.4038/sljr.v4i1.71

Keywords: occult breast carcinoma, orbital metastasis, ptosis, cutaneous nodules, hypothyroidism

\section{Introduction}

Although, breast carcinoma is a known cause of orbital metastases, these usually present after diagnosing the primary breast carcinoma. We present a case of a 55 year old female who presented with ptosis due to retro-orbital deposits as the first manifestation of an occult lobular carcinoma of breast. This case highlights the importance of knowing unusual manifestations of common malignancies. As she was also diagnosed of having chronic thyroiditis and hypothyroidism, it adds on to the association between benign thyroid diseases and breast carcinoma.

\section{Case report}

A 55 year old previously well female presented with ptosis of the right eye for three months duration. It was painless and was of gradual onset. Apart from ptosis she had felt heaviness of the eye. She did not have any visual defects. She had been having lethargy and fatiguability for one month duration. She was also having increased sleepiness. Ophthalmologic examination revealed no other abnormality. She was investigated with clinical suspicion of myasthenia gravis. She underwent a CT scan of the thorax, which revealed no abnormality. Her nerve conduction studies were normal.

During the same period she developed skin nodules in her left loin region and in the anterior abdominal wall. Ultrasound scan of

Corresponding author: M.C Wettasinghe, < chamimw003@yahoo.com>

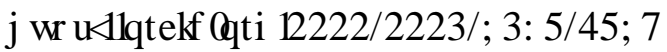




the skin nodules showed ill defined, low echogenic lesions.in the subcutaneous tissue plane. Both loin and anterior abdominal wall nodules were biopsied under ultrasound guidance. Biopsy revealed metastatic deposits of adenocarcinoma of breast origin.

Subsequently she underwent mammogram and ultrasound scan of the breasts. Mammogram revealed an ill defined focal asymmetric density in left upper outer quadrant with spiculated margins and subtle architectural distortion (Fig. 1). Lesion measured $15 \mathrm{~mm}$ in widest diameter. No microcalcifications were seen. There were bilateral axially lymphadenopathy with altered architecture.

Breast sonography revealed a subtle low echogenic area at 1:00 o'clock position, in inner zone, measuring $8 \times 4 \times 3 \mathrm{~mm}$ in size (Fig. 2). A thick echogenic halo was noted around the lesion. Bilateral axillary lymphadenopathy with altered architecture was also noted

Ultrasound guided biopsy of the breast lesion was performed. Histology revealed lobular carcinoma of breast, Nottingham Grade II. The malignancy was positive for both estrogen and progesterone receptors.
MRI of the orbits showed an ill-defined hypointense mass replacing the normal retro-orbital fat in right eye (Fig. 3,4). Both superior and inferior compartments of the eye were involved. It was extending into the lacrimal gland as well. Extra-orbital muscles showed abnormal signal intensities suggestive of muscle infiltration. The optic nerve and eye globe were spared. The lesion showed marked contrast enhancement. There was no extra-orbital extension of the mass. The cavernous sinuses was normal too. Left orbit was normal. No cerebral metastatic deposits were seen. Her pre-treatment $\mathrm{Tc}^{99 \mathrm{~m}} \mathrm{MDP}$ whole body bone scan revealed no bone metastasis (Fig 5).

The patient was investigated for thyroid disease and found to have high TSH. She was started on thyroxin. Ultrasound scan of the thyroid showed that thyroid gland volume was reduced with altered echopattern with relatively low echogenicity in both lobes (Fig. 6). There was moderately increased vascularity but no significant nodules. There were deep cervical adenopathy in both sides with the largest node in left side at level 2 measuring $15 \mathrm{~mm}$ in sagittal dimension. Appearances were compatible with chronic thyroiditis. 
She underwent a course of radiotherapy and chemotherapy. Post treatment whole body PET-CT showed a minimal reduction in size of the metastatic lesion in the right orbit which was metabolically active. A metabolically active metastatic lesion involving the outer cortex of the right occipital bone inferomedially, adjacent to the foramen magnum was also noted. She had mild chemotherapy induced pneumonitis. Same study revealed two small non FDG avid parenchymal nodules in the right lung. She is currently being followed up.

\section{Discussion}

Breast carcinoma is the most common malignancy in females with lobular carcinoma accounts for about $10 \%-15 \%$ of them ${ }^{1,2}$. When compared with ductal carcinoma, the sites of metastatic spread differ in lobular carcinoma. Although most breast carcinoma presents as a palpable breast lump, our patient present with unilateral ptosis and skin nodules, which represented metastatic deposits.

Breast carcinoma infrequently metastasizes to the orbit and most of the time this occurs after the primary tumor is diagnosed ${ }^{3,4}$. Overall prevalence of orbital deposits in breast carcinoma is around $0.2 \% .^{4}$ Orbital metastasis from occult breast carcinoma is even rarer. As stated in According to literature most metastatic deposits are unilateral. This rare presentation lead to the initial working diagnosis of myasthenia gravis in this patient, however subsequent development of skin nodules expedited the correct diagnosis.

In contrast to ductal carcinoma where the common sites of metastatic disease are lung, bones and liver, lobular carcinoma spreads to retroperitoneum, GI tract and, peritoneum. But these common sites was not seen in our patient ${ }^{5}$.

In our patient the thyroid disease and breast carcinoma were diagnosed concurrently. This contributes to the recent findings of an association of breast carcinoma with benign thyroid diseases. Studies conducted in Italy, have found that the overall prevalence of thyroid disorders is increased in patients with breast cancer, and thyroid autoimmune disorders, especially Hashimoto's thyroiditis, account to a large extent for the increased prevalence of thyroid disease in patients with breast cancer. They have also stated that thus is independent from the ER and PR status of the primary tumor. Similar association was found in our patient. But as 
stated in the above mentioned study, this association needs further evaluatoin ${ }^{6,7}$.

This case highlights the importance of keeping high degree of suspicion of uncommon manifestations of common malignancies, like breast carcinoma to avoid unnecessary delay in arriving at the correct diagnosis. This case further adds to the recent findings of breast association of thyroid disease with breast carcinoma.

\section{References:}

1. Raap M, Antonopoulos W, Dämmrich M, Christgen H, Steinmann D, Länger F, Lehmann U, Kreipe H, Christgen M. High frequency of lobular breast cancer in distant metastases to the orbit. Cancer medicine. 2015 Jan 1;4(1):104-11.

2. Arrangoiz R, Papavasiliou P, Dushkin H, Farma JM. Case report and literature review: Metastatic lobular carcinoma of the breast an unusual presentation. International journal of surgery case reports. 2011 Jan 1;2(8):301-5.

3. Francone E, Murelli F, Paroldi A, Margarino C, Friedman D. Orbital swelling as a first symptom in breast carcinoma diagnosis: a case report.
Journal of medical case reports. 2010 Dec;4(1):211.

4. Gupta S, Bhatt VR, Varma S. Unilateral orbital pain and eyelid swelling in a 46year-old woman: orbital metastasis of occult invasive lobular carcinoma of breast masquerading orbital pseudotumour. BMJ case reports. 2011 Jan 1;2011:bcr1220103580.

5. Sobinsky JD, Willson TD, Podbielski FJ, Connolly MM. Unusual metastatic patterns of invasive lobular carcinoma of the breast. Case reports in oncological medicine. 2013;2013.

6. Giani CL, Fierabracci PA, Bonacci RO, Gigliotti AG, Campani DA, De Negri FE, Cecchetti DE, Martino EN, Pinchera AL. Relationship between breast cancer and thyroid disease: relevance of autoimmune thyroid disorders in breast malignancy. The Journal of Clinical Endocrinology \& Metabolism. 1996 Mar 1;81(3):990-4.

7. Muller I, Pinchera A, Fiore E, Belardi V, Rosellini V, Giustarini E, Giani C. High prevalence of breast cancer in patients with benign thyroid diseases. Journal of endocrinological investigation. 2011 May 1;34(5):349-52. 


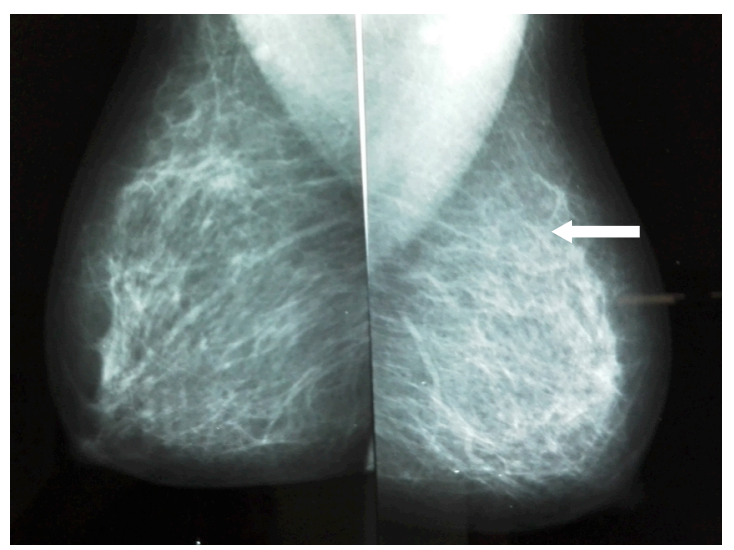

Figure 1: Mammogram -white arrow demonstrating ill defined focal asymmetric density in the left upper outer quadrant

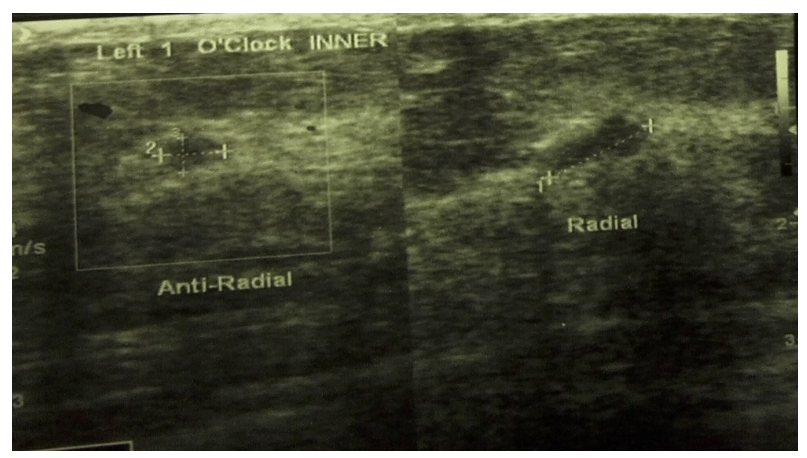

Figure 2: Left UOQ subtle low echogenic abnormality

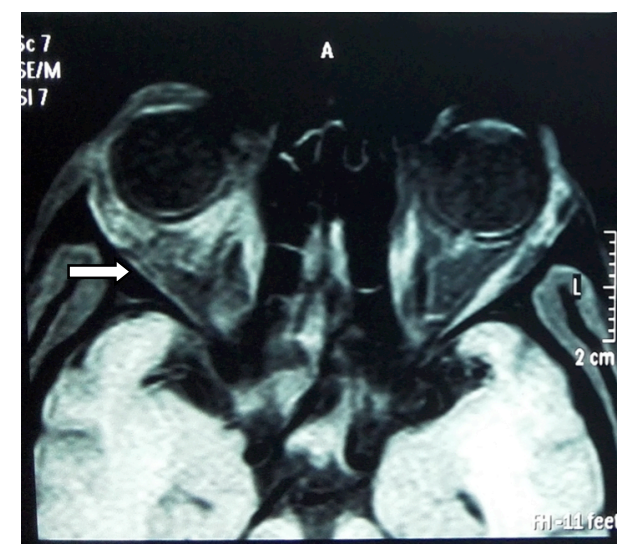

Figure 3:T1W MRI of orbits -

hypointense mass (white arrow) occupying the right side retro-orbital orbital space 


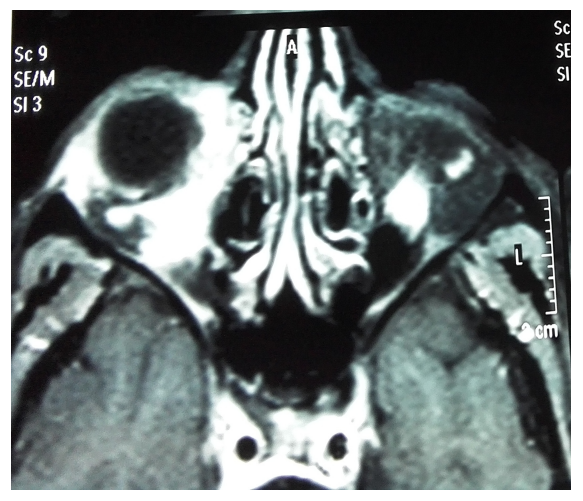

Figure 4: Contrast enhanced T1W MRI of orbits demonstrating avid contrast enhancement of the retroorbital mass

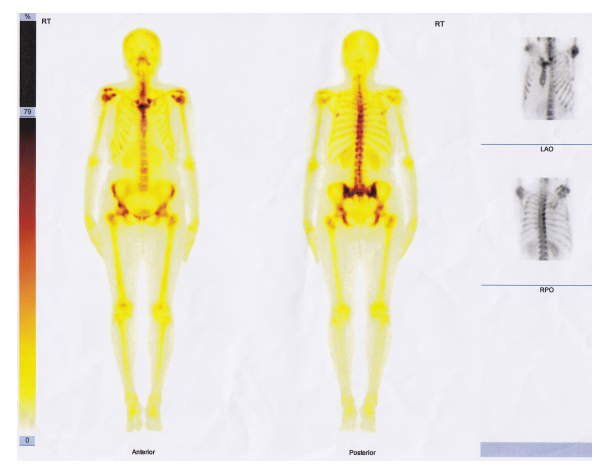

Figure 5: Negative bone scan

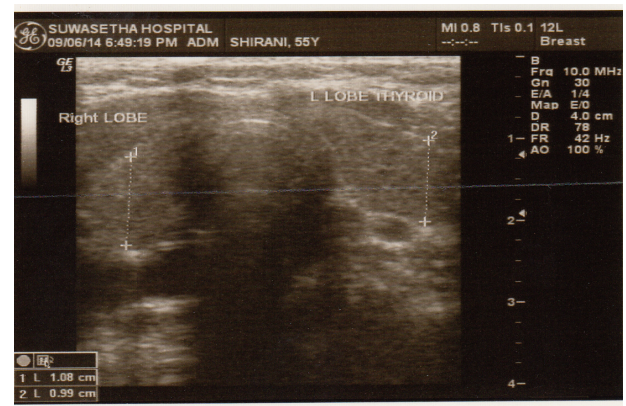

Figure 6: Thyroid sonography

demonstrating atrophied gland with coarse echo pattern 